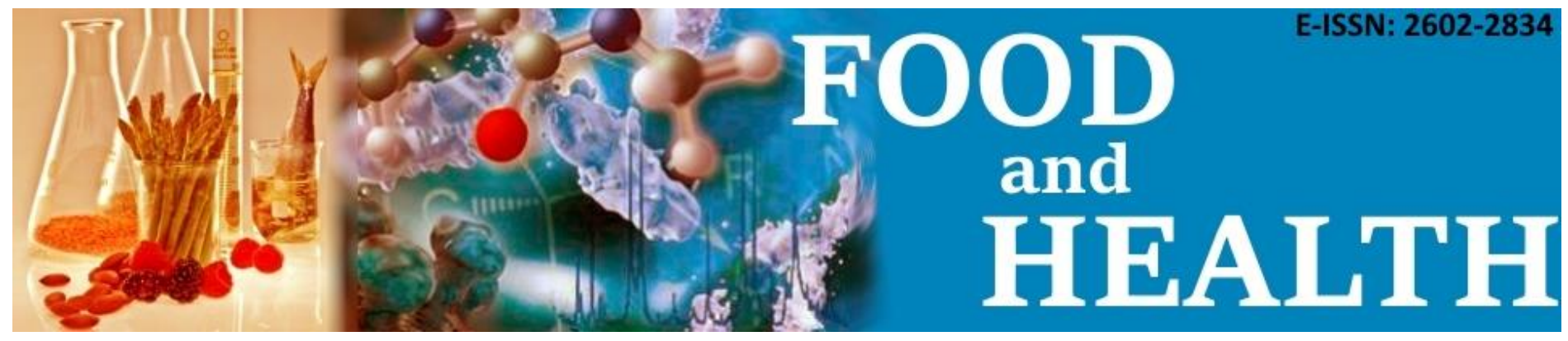

\title{
SIMULTANEOUS DETERMINATION OF BENZOIC ACID AND SORBIC ACID IN FOOD PRODUCTS BY CAPILLARY ELECTROPHORESIS
}

\section{Nevin Öztekin î}

\section{Cite this article as:}

Öztekin, N. (2018). Simultaneous Determination of Benzoic Acid and Sorbic Acid in Food Products by Capillary Electrophoresis. Food and Health, 4(3), 176-182. DOI: $10.3153 / \mathrm{FH} 18018$

Istanbul Technical University, Department of Chemistry, Maslak, 34469 Istanbul, Turkey

Submitted: 12.08 .2017

Accepted: 12.12.2017

Published online: 13.03.2018

Correspondence:

Nevin ÖZTEKİN

E-mail: noztekin@itu.edu.tr

\begin{abstract}
In this work, a rapid and easy capillary electrophoretic method for simultaneous determination of benzoic acid and sorbic acid in food samples using direct UV detection was reported. The separation of acids was achieved in fused silica capillary at $28 \mathrm{kV} .20 \mathrm{mM}$ borate buffer at pH 9.3 was used as running buffer; cinnamic acid was chosen as internal standard for quantification. Under the optimal separation conditions benzoic acid and sorbic acid were detected in $3.1 \mathrm{~min}$. The linear ranges were between $0.005-0.4 \mathrm{mM}$. The correlation coefficients for each calibration curve were calculated as 0.999. The reproducibility of peak area and migration time for each acid were less than $3 \%$ (R.S.D.). The limits of detection were found $0.405 \mu \mathrm{g} / \mathrm{mL}$ for benzoic acid and $0.415 \mu \mathrm{g} / \mathrm{mL}$ for sorbic acid. The limits of quantification were $1.35 \mu \mathrm{g} / \mathrm{mL}$ and $1.38 \mu \mathrm{g} / \mathrm{mL}$ respectively.
\end{abstract}

Keywords: Capillary electrophoresis, Benzoic acid, Sorbic acid, Foods 


\section{Introduction}

Benzoic acid (E210) and sorbic acid (E200) are widely used as food additives to delay the microbiological contamination due to action of bacteria, yeasts and moulds in various foods. They are supposed as usually reliable preservatives. If food products contain high concentrations of these acids, they may damage to health of consumers due to causing allergic reactions. Like the other countries, the use of these additives have been restricted according to Turkish Food Codex regulations of Agriculture Ministry of Turkey. In order to protect human health, determination of maximum permitted amounts of these additives in food products is very important. Consequently, easy and reliable analysis methods for detection of these additives in foodstuffs is required for food safety. Several analytical methods are available for the determination of additives in foods like UV spectrometry (Marsili, et al., 2003; Naseri, et al. 2017) and GC (Ochiai et al., 2002; Abedi et al. 2014). Both of these methods require comprehensive sample preparation and relatively long analysis time. HPLC is most commonly used method for the determination of these additives in foods and beverages (Mota et al., 2003; Pylypiw Jr \& Grether, 2000; Tfouni \& Toledo, 2002; Zor et al. 2016; Petanovska-Ilievska et al. 2017). HPLC generally needs the consumption of large amounts of organic solvents during the analysis and extensive sample preparation step, including solid phase extraction.

Capillary electrophoresis (CE) is an analytical method that offer high separation efficiency and is ensuring a fast separation for the assay of several species. CE method has some advantages such as fast analysis time, low or no organic solvent consumption and little injection volume. A matrix effect in sample can be eliminated with short and simple sample pretreatment. The capillary between runs is quickly washed with running buffer. Therefore it is a convenient analysis technique for using in the analysis of foods contained complex matrices.

The inhibitory action of weak acids is classically believed to be due to the undissociated compounds. Because, the uncharged, undissociated state of the acid is freely passed through the plasma membrane and is thus ensured to enter the cell (Brul \& Coote, 1999). $\mathrm{pK}_{\mathrm{a}}$ values of benzoic and sorbic acid are smaller than $\mathrm{pH} 7$ (4.19 and 4.76 respectively). The activity of them in foods that has value of low $\mathrm{pH}$ increase. These acids are used as a substitute for each other. Nevertheless, we observed the mixtures of benzoic and sorbic acid are used in some food products in Turkey. While several of the CE reports demonstrated the possibilities for simultaneous determination of benzoic and sorbic acids (Boyce, 1999; Frazier et al., 2000; Kuo \& Hsieh, 1997; Lin et al., 2000; Pant \& Trenerry, 1995; Waldron \& Li,
1996; Han et al., 2008; Hsu et al., 2014; Sun et al., 2014; Li et al., 2015; Aung \& Pyell 2016), only a few of them reported benzoic and sorbic acids together in real samples.

This study presents an application for the quantification of benzoic and sorbic acid in the food samples in the Turkish markets by using CE method. Before the injection processes, any pretreatment procedure was not carried out for the food samples.

\section{Materials and Methods}

\section{Instrumentation}

For separations and determinations, an Agilent CE system (Waldbronn, Germany) equipped with a diode array detector was used. Agilent Chem Station software performed all system control and data processing. In separations, $50 \mu \mathrm{m}$ I.D. fused-silica capillary (Polymicro Technologies, Phoenix, AZ, USA) with $46 \mathrm{~cm}$ total length and $38 \mathrm{~cm}$ effective length was used. The capillary temperature was set at $25^{\circ} \mathrm{C}$. The separations were performed at voltage of $28 \mathrm{kV}$. The injection of samples was made at pressure 50 mbar for $4 \mathrm{~s}$. Before injections at the beginning of every day the capillary was conditioned with $0.1 \mathrm{M} \mathrm{NaOH}$ solution for $15 \mathrm{~min}$, deionized water for $5 \mathrm{~min}$ and running buffer for $10 \mathrm{~min}$. between runs 1 min of flushing with running buffer was performed. A Metrohm 654 Digital $\mathrm{pH}$ Meter was used for $\mathrm{pH}$ measurements. All solutions were prepared with deionized water obtained from an Elgacan C114 filtration system.

\section{Chemicals}

Benzoic acid, sorbic acid, and cinnamic acid were purchased from Fluka (Fluka AG, Buchs, Switzerland). Sodium borate was obtained from Merck (Darmstadt, Germany). Other chemicals used were of analytical reagent grade. The food samples were from local market.

\section{Standards}

Stock solutions of benzoic acid, sorbic acid, and cinnamic acid were prepared by dissolving in water, and stored at $4^{\circ} \mathrm{C}$.

\section{Samples}

After solid samples were homogenized, 1-2 g samples were weighed in a beaker. $15 \mathrm{~mL}$ of deionized water was added to samples and stirred during $30 \mathrm{~min}$. The volume of sample was diluted to $20 \mathrm{~mL}$. The solutions were centrifuged a 7000 rpm for $5 \mathrm{~min}$ and filtered through a $0.45 \mu \mathrm{m}$ syringe filter. Internal standard solution (cinnamic acid) was added to filtrate and the sample solution volume was diluted with water. The completed solution was injected directly into the capillary. Liquid food samples were prepared by dilution with water. 


\section{Results and Discussion}

In $\mathrm{CE}$ analysis the electroosmotic flow (EOF) plays an important role. The magnitude of the EOF can influence the resolution and separation efficiency. As we all know, the EOF can be determined by net surface charge of the inner surface of capillary. At higher $\mathrm{pH}$ (usually alkaline), faster EOF are obtained due to the full ionization of silanol groups. The concentration and $\mathrm{pH}$ of buffer are the two important parameters for adjusting the EOF and the electrophoretic mobility. Therefore the separation buffer affects the migration time and the resolution between analytes. The ionization degree of an organic acid also depends on choice of the buffer. At the same time the $\mathrm{pH}$ of the buffer influences ionization degree and separation of organic acids. Simultaneous migrations of benzoic acid, sorbic acid, and cinnamic acid were tested in the three different separation buffer namely; borate, phosphate and Tris in the $\mathrm{pH}$ range of 7 and 9.5. Their $\mathrm{pK}_{\mathrm{a}}$ values are 4.19, 4.76 and 4.27, respectively and in this $\mathrm{pH}$ range all acids are in higher ionization degrees. Thus they can be separated as anions in alkaline media.

When borate buffer was used as the separation media, peak heights and peak areas were observed as better than the peaks in the other two buffers, and also migration times were shorter. Therefore borate was selected as the running buffer. Dilute buffer solutions are desirable to shorten migration time. However, it is important that buffer solutions have sufficient buffer capacity to maintain a fixed $\mathrm{pH}$. Inadequate buffer capacity may result in the poor resolution. The effect of borate concentration was studied within the range 10-50 mM. At higher borate concentrations (>50 mM) longer migration time and broader peak were obtained. Optimum peak symmetry and peak area were achieved with 20 $\mathrm{mM}$ borate.

When the running voltages is increased the efficiency of the separation improves and the migration time shortens. In the higher running voltage, the efficiency of the separation decrease due to the Joule heating. On the other hand, the reproducibility and the resolutions between acids becomes worse if the running voltage decreases. In this study a voltage of $28 \mathrm{kV}$ was selected as the best separation voltage with acceptable compromise between adequate resolution and short migration time.
In CE the high amounts of matrix components in the samples affect the peak shapes of analytes has generally low concentrations. In order to check the matrix effect on the peak shapes, the real samples was injected to the borate buffer with increasing concentrations. It was not observed a distortion in the peak shapes in the small borate concentrations. Since the matrix effect was not observed in the highly diluted real samples, all separations were performed in the $20 \mathrm{mM}$ borate buffer at $\mathrm{pH} 9.3$, considering the fast separation times.

Figure 1 shows the absorption spectra of the analytes and internal standard in the same concentration level. In Figure 2 the peak areas of benzoic acid, sorbic acid, and cinnamic acid were given at the different wavelengths varying from 210 to $270 \mathrm{~nm}$. According to peak areas $220 \mathrm{~nm}$ can be selected for the simultaneous screening of benzoic acid, sorbic acid, and cinnamic acid peaks. In this study because of using a diode array detector for the detection, benzoic acid and sobic acid was dedected different wavelengths respectivly $220 \mathrm{~nm}$ and $260 \mathrm{~nm}$.

The electropherogram of standard mixture of acids at optimum experimental conditions are shown in Figure 3a. The samples were injected directly to the borate buffer and all acids were detected in about $3.0 \mathrm{~min}$.

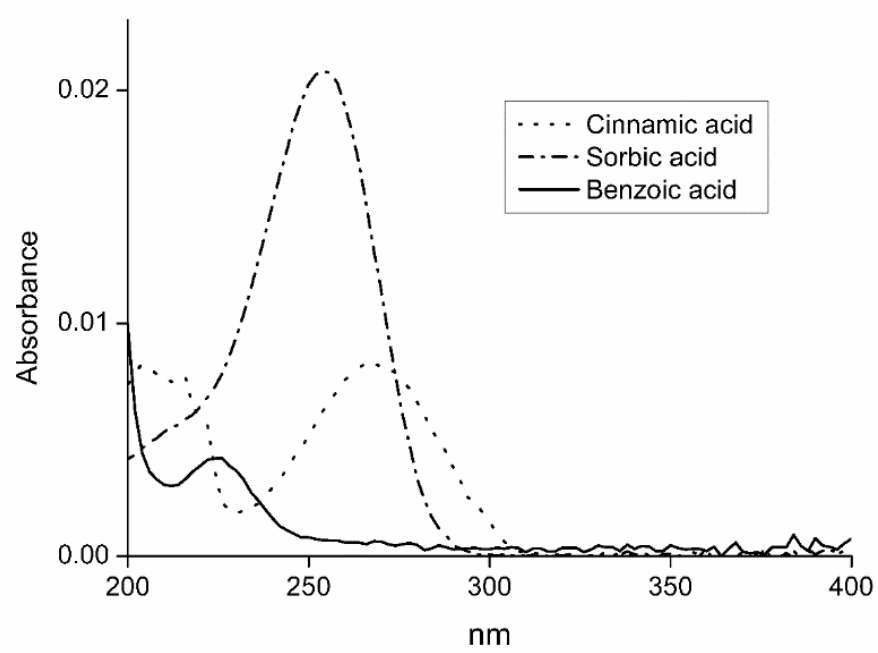

Figure 1. Spectra of benzoic, sorbic and cinnamic acid 


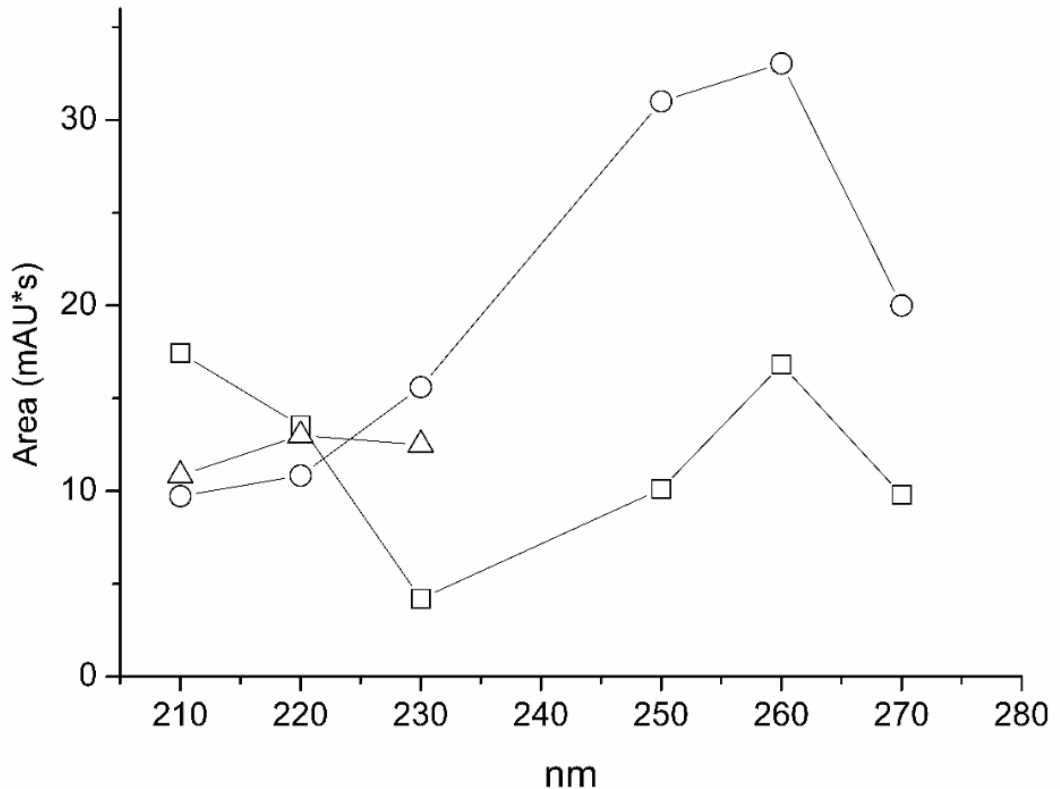

Figure 2. The effect of the wavelength on the peak areas of benzoic acid $(\triangle)$, sorbic acid $(O)$ and cinnamic acid $(\square)$. Running buffer, $20 \mathrm{mM}$ Borate, $\mathrm{pH} 9.3$; injection, $4 \mathrm{~s}$ at 50 mbar; run voltage, $28 \mathrm{kV}$

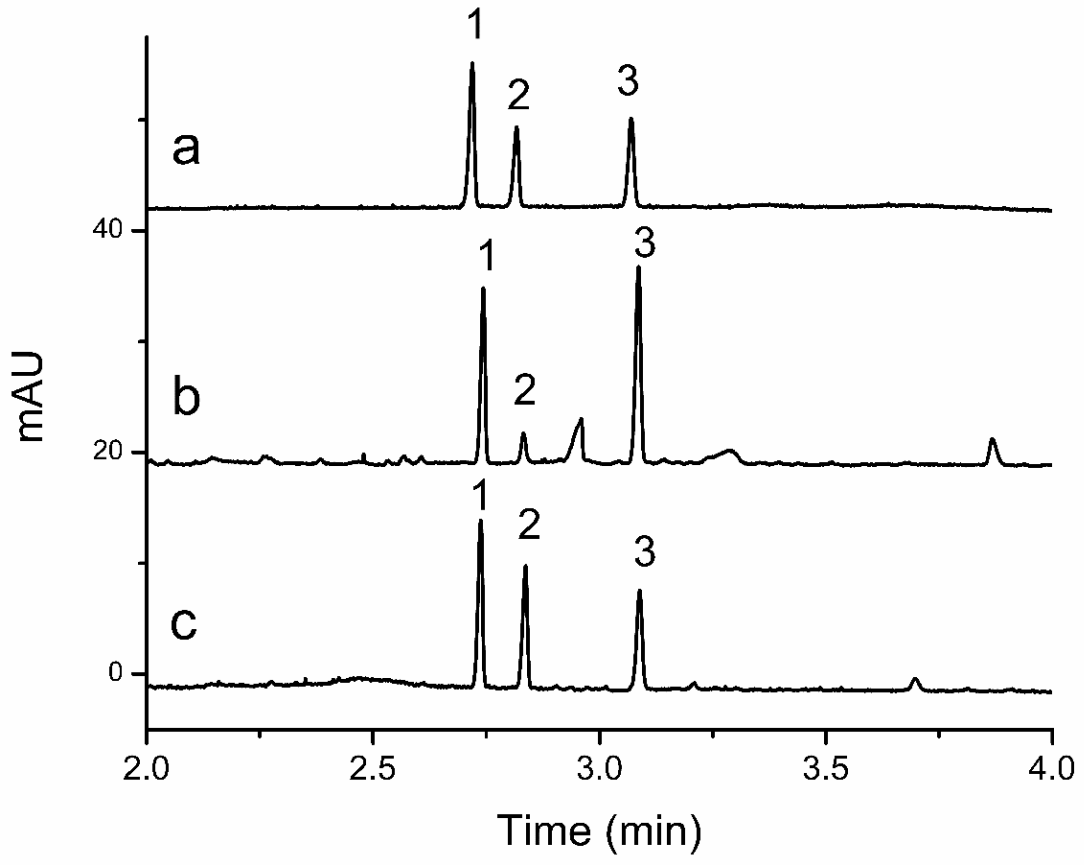

Figure 3. Electropherograms of acids. (a) standard solution; (b) ketchup sample and (c) chestnut sweet sample. Peaks: (1) cinnamic acid, (2) sorbic acid, (3) benzoic acid. Experimental conditions: capillary, fused-silica $50 \mu \mathrm{m}$ i.d. and $46 \mathrm{~cm}$ (38 cm effective length); background electrolyte, $20 \mathrm{mM}$ Borate, $\mathrm{pH}$ 9.3; injection, $4 \mathrm{~s}$ at $50 \mathrm{mbar}$; temperature $25^{\circ} \mathrm{C}$; running voltage $28 \mathrm{kV}$; detection signal $=220 \mathrm{~nm}$ 


\section{Method Validation}

\section{Linearity}

The calibration curves were obtained between the peak area ratios $\left(\mathrm{A}_{\text {analyte}} / \mathrm{A}_{\text {internal standard }}\right)$ and the concentrations of the acids. The calibration curves for benzoic acid and sorbic acid showed linear ranges between 0.005 and $0.4 \mathrm{mM}$. Linear regression equations for benzoic acid and sorbic acid were obtained as $\mathrm{y}=8.14010^{-1} \mathrm{x}-9.55810^{-4}$ and $\mathrm{y}=6.47410^{-1}$ $\mathrm{x}-3.45910^{-3}$ respectively. The correlation coefficients values were greater than 0.999 .

Limit of detection
Limits of detection (LOD) were calculated as three times the baseline noise $(\mathrm{S} / \mathrm{N}=3)$. The LOD value was $0.405 \mu \mathrm{g} / \mathrm{mL}$ for benzoic acid and $0.415 \mu \mathrm{g} / \mathrm{mL}$ for sorbic acid. Limits of quantification (LOQ) ( $\mathrm{S} / \mathrm{N}=10)$ was $1.35 \mu \mathrm{g} / \mathrm{mL}$ and 1.38 $\mu \mathrm{g} / \mathrm{mL}$ respectively.

\section{Reproducibility}

The reproducibility of the CE method was determined by analyzing two real samples, Ketchup and Chestnut candy. \% RSD values of migration times and peak areas were calculated for intra-day and inter-day. Intra-day and inter-day precision were calculated seven successive injections in a day and three injections in different days, respectively. The results are given in Table 1.

Table 1. Reproducibilities of the method for two sample. (I) Ketchup, (II) Chestnut sweet

\begin{tabular}{|c|c|c|c|c|c|c|}
\hline & \multicolumn{2}{|c|}{ Intraday RSD\% } & \multicolumn{2}{|c|}{ Inter day RSD\% } & \multicolumn{2}{|c|}{$\begin{array}{c}\text { mg anion/kg sample } \\
\text { RSD \% }\end{array}$} \\
\hline & Migration time & Peak area & Migration time & Peak area & Inter day & Intra day \\
\hline & & & Sample 1 & & & \\
\hline Benzoic & 0.213 & 0.375 & 0.645 & 1.234 & 0.364 & 1.376 \\
\hline Sorbic & 0.194 & 0.877 & $\begin{array}{l}0.724 \\
\text { Sample } 2\end{array}$ & 1.582 & 0.956 & 2.753 \\
\hline Benzoic & 0.0741 & 0.638 & 0.445 & 2.398 & 0.744 & 1.942 \\
\hline Sorbic & 0.0697 & 0.559 & 0.421 & 2.179 & 0.601 & 1.894 \\
\hline
\end{tabular}

Table 2. Recovery of the method for two samples. (I) Ketchup, (II) Chestnut sweet

\begin{tabular}{ccccc}
\hline Added amount & \multicolumn{2}{c}{ Sample (I) Recovery (\%) } & \multicolumn{2}{c}{ Sample (II) Recovery (\%) } \\
\cline { 2 - 5 } & Benzoic acid & Sorbic acid & Benzoic acid & Sorbic acid \\
\hline $0.03 \mathrm{mM}$ & 97 & 98 & 104 & 101 \\
$0.06 \mathrm{mM}$ & 98 & 103 & 101 & 102 \\
$0.10 \mathrm{mM}$ & 103 & 100 & 99 & 101 \\
\hline
\end{tabular}

Table 3. Results for the determination of benzoic acid and sorbic acid in various food products

\begin{tabular}{lcc}
\hline Sample & Benzoic acid $(\mathrm{mg} / \mathrm{kg}$ or $\mathrm{mg} / \mathrm{L})$ & Sorbic acid $(\mathrm{mg} / \mathrm{kg}$ or $\mathrm{mg} / \mathrm{L})$ \\
\hline Lemon sauce & $4.36 \pm 0.12$ & $235.5 \pm 0.9$ \\
Pickle & $126.9 \pm 1.1$ & $127.3 \pm 0.9$ \\
Fruit juice & $234.9 \pm 0.9$ & $178.5 \pm 0.7$ \\
Ketchup & $463.2 \pm 1.0$ & $75.3 \pm 1.8$ \\
Chestnut sweet & $200.5 \pm 1.9$ & $251.5 \pm 1.9$ \\
Olive paste & $13.1 \pm 1.7$ & $600.7 \pm 1.5$ \\
Pepper paste & $495.5 \pm 1.8$ & $10.5 \pm 0.4$ \\
Pepper sauce & $182.8 \pm 1.6$ & $188.5 \pm 1.2$ \\
Cake & n.d. & $600.7 \pm 2.0$ \\
\hline
\end{tabular}

n.d.: not detected 


\section{Recovery}

The accuracy of method was evaluated with two methods: (1) The peak purity was analyzed with diode array detector and (2) the known amounts of acid standards were added to food samples. Table 2 shows the recovery results for three concentration levels and for two food samples.

\section{Application to Food Samples}

The benzoic acid and sorbic acid in nine food products were quantified by using the CE method. Figures $3 b$ and $3 c$ illustrate the electropherograms of two food samples. In optimized conditions acids were detected within about $3.0 \mathrm{~min}$. Amounts of preservatives determined in each sample were listed in Table 3. As seen from the electropherograms, there are unknown peaks in this region, but peaks of acids and internal standard are seen clearly. All food samples contain sorbic acid. In only one food sample benzoic acid was not detected. The amount of determined benzoic acid in food samples was between 4.36 and $495.5 \mathrm{mg} / \mathrm{kg}$ or $\mathrm{mg} / \mathrm{L}$. The content of sorbic acid in food samples ranged from 10.5 to $600.7 \mathrm{mg} / \mathrm{kg}$ or $\mathrm{mg} / \mathrm{L}$. The obtained data indicate that the preservatives content in foods were under permitted maximum levels.

\section{Conclusion}

In this work a simple, easy and reliable CE method was described for the simultaneous determination of benzoic and sorbic acid in various food products. The linearity of calibration curves was very good ( $r>0.999)$ and the reproducibility of method were also satisfactory (RSD $<3 \%$ ). Method contain both short sample preparation time and short analysis time $(\sim 3.0 \mathrm{~min})$. The matrix of samples did not interfere the analysis of analytes. In addition it contain low electrolyte and sample consumption. The silica capillaries are cheaper than chromatographic columns, easily washed between runs. Therefore the proposed CE method is used both as alternative method to HPLC for the analysis of additives in food products and routine analysis of various food for samples benzoic and sorbic acid.

\section{References}

Abedi, A.S., Mohammadi, A., Azadniya, E., Mortazavian, A.M., Khaksar, R. (2014). Simultaneous determination of sorbic and benzoic acids in milk products using an optimised microextraction technique followed by gas chromatography. Food Additives and Contaminants Part A, 31, 21-28
Aung, H.P., Pyell, U. (2016). In-capillary derivatization with o-phthalaldehyde in the presence of 3-mercaptopropionic acid for the simultaneous determination of monosodium glutamate, benzoic acid, and sorbic acid in food samples via capillary electrophoresis with ultraviolet detection. Journal of Chromatography A, $1449,156-165$

Boyce, M.C. (1999). Simultaneous determination of antioxidants, preservatives and sweeteners permitted as additives in food by mixed micellar electrokinetic chromatography. Journal of Chromatography A, 847, 369375.

Brul, S., Coote, P. (1999). Preservative agents in foods Mode of action and microbial resistance mechanisms. International Journal of Food Microbiology, 50, 1-17.

Frazier, R.A., Inns, E.L., Dossi, N., Ames, J.M., Nursten, H.E. (2000). Development of a capillary electrophoresis method for the simultaneous analysis of artificial sweeteners, preservatives and colours in soft drinks. Journal of Chromatography A, 876, 213-220.

Han, F., He, Y.Z., Li, L., Fu, G.N., Xie, H.Y., Gan, W.E. (2008). Determination of benzoic acid and sorbic acid in food products using electrokinetic flow analysis-ion pair solid phase extraction-capillary zone electrophoresis. Analytica Chimica Acta, 618, 79-85.

Hsu, S.H., Hu, C.C., Chiu, T.C. (2014). Online dynamic pH junction-sweeping for the determination of benzoic and sorbic acids in food products by capillary electrophoresis. Analytical and Bioanalytical Chemistry, 406, 635-641.

Kuo, K.L., Hsieh, Y.Z. (1997). Determination of preservatives in food products by cyclodextrin modified capillary electrophoresis with multiwavelength detection. Journal of Chromatography A, 768, 334-341.

Li, B., Li, Y.K., Wang, X.Y., Wang, F., Wang, X., Wang, Y.F., Meng, X.J. (2015). Simultaneous separation and determination of organic acids in blueberry juices by capillary electrophoresis- electrospray ionization mass spectrometry. Journal of Food Science and Technology, 52, 5228-5235.

Lin, Y.H., Chou, S.S., Sheu, F., Shyu, Y.T. (2000). Simultaneous determination of sweeteners and preservatives in preserved fruits by micellar electrokinetic capillary 
chromatography. Journal of Chromatographic Science, 38, 345-352.

Marsili, N., Sobrero, M.S., Goicoechea, H.C. (2003). Spectrophotometric determination of sorbic and benzoic acids in fruit juices by a net analyte signal-based method with selection of the wavelength range to avoid nonmodelled interferences. Analytical and Bioanalytical Chemistry, 376, 126-133.

Mota, F.J.M., Ferreira, I.M.P.L.V., Cunha, S.C., Beatriz, M., Oliveira, P.P. (2003). Optimization of extraction procedures for analysis of benzoic and sorbic acids in foodstuffs. Food Chemistry, 82, 469-473.

Naseri, A., Farahmand, F., Sheykhizadeh S., Tabibiazar M., (2017). Coupling of air-assisted liquid-liquid microextraction method with partial least squares for simultaneous spectrophotometric determination of some preservatives. Journal of the Iranian Chemical Society, 14, 643-653.

Ochia, N., Sasamoto, K., Takino, M., ·Yamashita, S., Daishima, S., Heiden, A.C., Hoffmann, A. (2002). Simultaneous determination of preservatives in beverages, vinegar, aqueous sauces, and quasi-drug drinks by stirbar sorptive extraction (SBSE) and thermal desorption GC-MS. Analytical Bioanaytical Chemistry, 373, 5663

Pant, I., Trenerry, C. (1995). The determination of sorbic acid and benzoic acid in a variety of beverages and foods by micellar electrokinetic capillary chromatography. Food Chemistry, 53 219-226.
Petanovska-Ilievska, B., Velkoska-Markovska, L., Jankulovska, M.S. (2017). Development of reverse-phase high-performance liquid chromatography method for simultaneous determination of sodium benzoate and potassium sorbate in beverages. Acta Chromatographica, 29, 345-358.

Pylypiw, Jr. H.M., Grether, M.T. (2000). Rapid high-performance liquid chromatography method for the analysis of sodium benzoate and potassium sorbate in foods. Journal of Chromatography A, 883, 299-304

Sun, B., Qi, L., Wang, M. (2014). Determination of preservatives in soft drinks by capillary electrophoresis with ionic liquids as the electrolyte additives. Journal of Separation Science, 37, 2248-2252.

Tfouni, S.A.V., Toledo, M.C.F. (2002). Determination of benzoic and sorbic acids in Brazilian food. Food Control, 13, 117-123.

Waldron, K.C., Li, J. (1996). Investigation of a pulsed-laser thermo-optical absorbance detector for the determination of food preservatives separated by capillary electrophoresis. Journal of Chromatography B, 683, 47-54.

Zor, Ş.D., Aşç1, B., Dönmez, Ö.A., Küçükkaraca, D.Y. (2016). Simultaneous determination of potassium sorbate, sodium benzoate, quinoline yellow and sunset yellow in lemonades and lemon sauces by HPLC using experimental design. Journal of Chromatographic Science, 54, 952-957. 